\title{
A two-step surgical approach combining sternotomy and subsequent thoracotomy for locally advanced lung cancers requiring both right upper lung resection and superior vena cava reconstruction
}

\author{
Han-Yu Deng ${ }^{1,2 \#}$, Chang-Long Qin ${ }^{1}$, Xiao-Ming Qiu ${ }^{1 \#}$, Xiao-Jun Tang ${ }^{1 \#}$, Da-Xing Zhu ${ }^{1}$, Qinghua Zhou ${ }^{1}$ \\ ${ }^{1}$ Lung Cancer Center, ${ }^{2}$ Department of Thoracic Surgery, West China Hospital of Sichuan University, Chengdu 610041, China \\ Contributions: (I) Conception and design: Q Zhou; (II) Administrative support: Q Zhou; (III) Provision of study materials or patients: HY Deng, \\ CL Qin; (IV) Collection and assembly of data: HY Deng, XM Qiu; (V) Data analysis and interpretation: XM Qiu, XJ Tang, DX Zhu; \\ (VI) Manuscript writing: All authors; (VII) Final approval of manuscript: All authors. \\ "These authors contributed equally to this work. \\ Correspondence to: Qinghua Zhou. Lung Cancer Center, West China Hospital of Sichuan University, No. 37 Guoxue Alley, Chengdu 610041, China. \\ Email: zhouqh135@163.com.
}

Background: Locally advanced lung cancers involving both right upper lung lobe and superior vena cava
(SVC) requiring both lung resection and SVC reconstruction are generally deemed unresectable. However,
previous evidence has proved that such patients could benefit from surgery if radical resection is achieved.
Generally, a hemi-clamshell approach is adopted to complete such resection. However, it has the limitation
of insufficient exposure of posterior mediastinum. Therefore, we introduced a two-step surgical approach
combining sternotomy and thoracotomy for such lung cancers. Methods: A two-step surgical approach combining median sternotomy and subsequent posterolateral thoracotomy, via which radical lobectomy with systematic lymphadenectomy and SVC reconstruction could be successfully achieved, was described.

Results: We have performed such surgery via the two-step surgical approach combining median sternotomy and subsequent posterolateral thoracotomy in five patients from January 2017 to March 2018. All those patients achieved radical resection of the lung cancer with lobectomy and systematic lymphadenectomy and SVC reconstruction with artificial blood vessels, and had an uneventful postoperative recovery without any major complications.

Conclusions: Our initial experience proved that this two-step surgical approach combining median sternotomy and subsequent posterolateral thoracotomy was safe and feasible for locally advanced lung cancers requiring both lung resection and SVC reconstruction.

Keywords: Locally advanced lung cancer; superior vena cava (SVC); radical resection; median sternotomy; posterolateral thoracotomy

Submitted Mar 24, 2018. Accepted for publication Jul 10, 2018.

doi: $10.21037 /$ jtd.2018.07.77

View this article at: http://dx.doi.org/10.21037/jtd.2018.07.77

\section{Introduction}

Locally advanced lung cancers invading great vessels present with a unique management challenge in clinical practice (1) and are commonly deemed as contraindication for surgery because of potential incomplete resection and high perioperative morbidity and mortality rates (2). However, for certain patients, radical resection could 


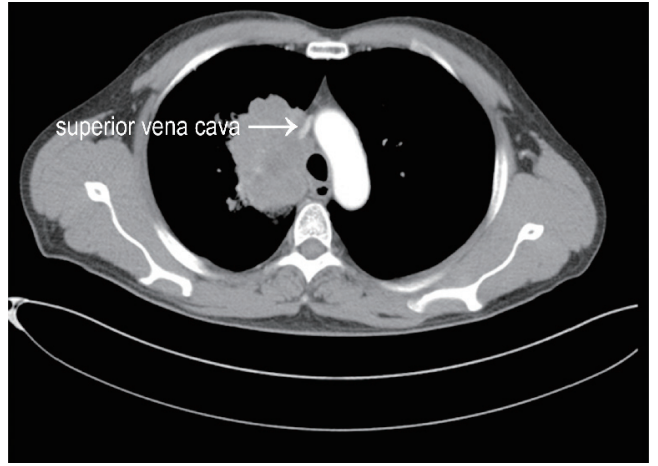

Figure $1 \mathrm{~A}$ mass was detected in the right thorax involving both upper right lobe and superior vena cava.
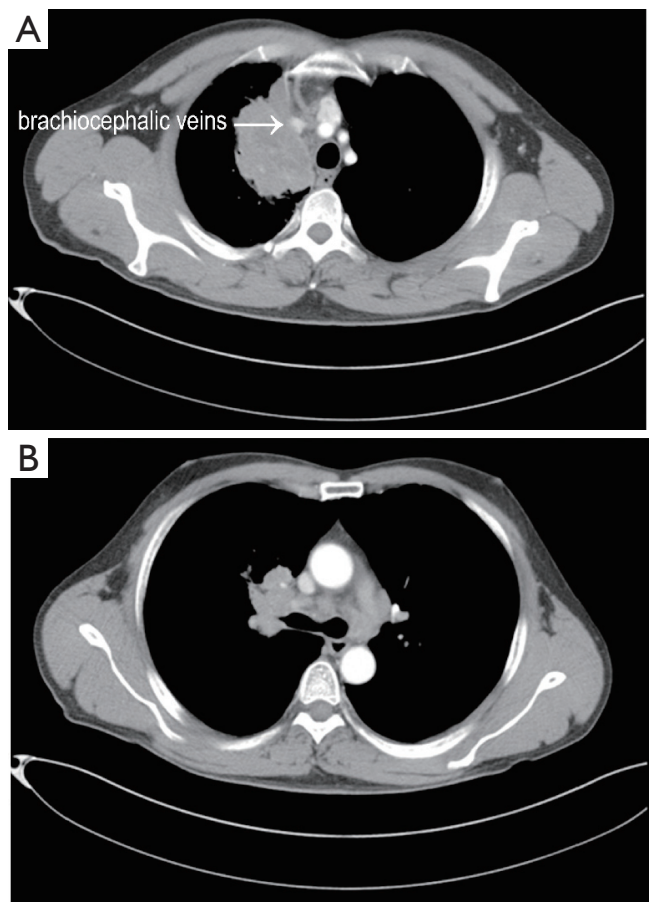

Figure 2 Invasion of the brachiocephalic veins (A) and enlarged ipsilateral and contralateral mediastinal lymph nodes (B) were also observed.

achieve long-term survival $(3,4)$. Therefore, surgery could be an option for certain patients if their tumors could be radically resected. For such lung cancers invading superior vena cava (SVC), usually a median sternotomy or lateral thoracotomy was applied for surgical resection (5). However, a median sternotomy has the limitation of insufficient access to the posterior mediastinum as well as difficulty in completing lung resection such as lobectomy and sleeve lobectomy, while a lateral thoracotomy has the limitation of insufficient access to the anterior mediastinum especially for accessing the left brachiocephalic vein. Therefore, a median sternotomy or lateral thoracotomy alone is not sufficient for resecting locally advanced lung cancers requiring both right upper lung resection and SVC reconstruction. Therefore, for such lung cancers, a hemiclamshell surgical approach consisting of a partial median sternotomy and anterolateral thoracotomy was generally adopted (6), providing an anterior view of the mediastinum and exposure of the apex of the chest (7). However, the hemi-clamshell approach also has several disadvantages, such as insufficient exposure of the posterior mediastinum and causing rib fracture $(7,8)$. Therefore, in order to achieve a radical resection of locally advanced lung cancers invading both right upper lung and SVC, we introduced a twostep surgical approach combining median sternotomy and subsequent posterolateral thoracotomy. Here we report this two-step surgical approach in a typical patient as follow.

\section{Methods}

\section{Surgical technique from a typical case report}

A 42-year-old man presented with severe cough and facial and neck swelling was admitted into our department. His computed tomography (CT) revealed a mass in the right thorax involving both upper right lung lobe and SVC (Figure 1). Moreover, invasion of the brachiocephalic veins (Figure $2 A$ ) and enlarged ipsilateral and contralateral mediastinal lymph nodes (Figure $2 B$ ) were also observed. This patient underwent radical resection of the mass with lobectomy and systematic lymphadenectomy with reconstruction of SVC via two-step surgical approachcombination of median sternotomy and subsequent posterolateral thoracotomy for easy and enough access to contralateral mediastinum and ipsilateral posterior mediastinum (Figure 3).

At first, the patient was placed at supine position and general anesthesia was administrated with a doublelumen endobronchial tube. And then an extracorporeal venous shunt was made connecting right internal jugular vein to the right femoral vein before surgery. After the extracorporeal venous shunt was established, the patient underwent median sternotomy (the first step), via which the left brachiocephalic vein was freed and cut off, and then it was reconnected to the right atrial appendage (Figure 4) with a 12-mm GORE-TEX ${ }^{\circledR}$ vascular graft (W.L. Gore and 


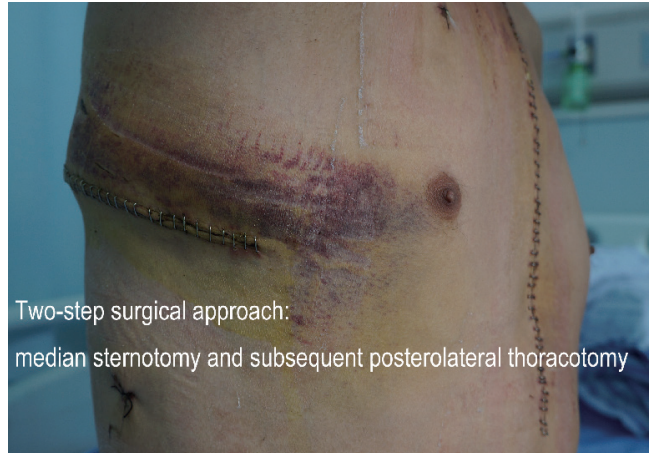

Figure 3 Demonstration of the two-step surgical approach: a median sternotomy and a subsequent posterolateral thoracotomy.
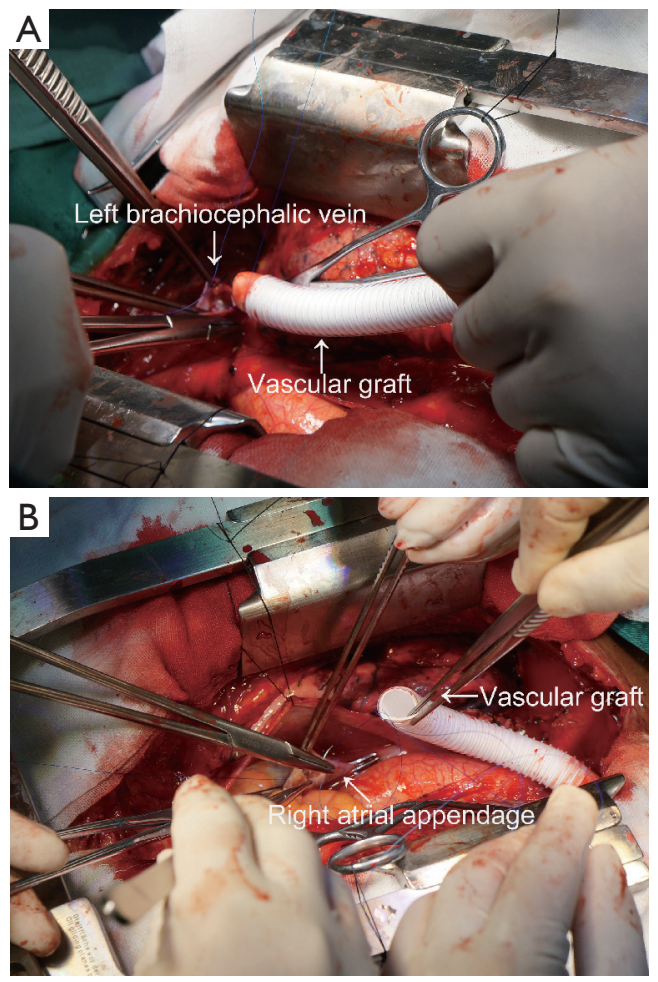

Figure 4 Demonstration of the first step via median sternotomy: (A) anastomosis between left brachiocephalic vein and vascular graft; (B) anastomosis between vascular graft and right atrial appendage.

Associates, AZ, USA) using 4-0 prolene sutures (Johnson \& Johnson) for continuous suturing. Then dissection of the left mediastinal lymph nodes (5\# and 6\#) was also achieved. Finally, the incision of median sternotomy was closed up in a standard fashion.

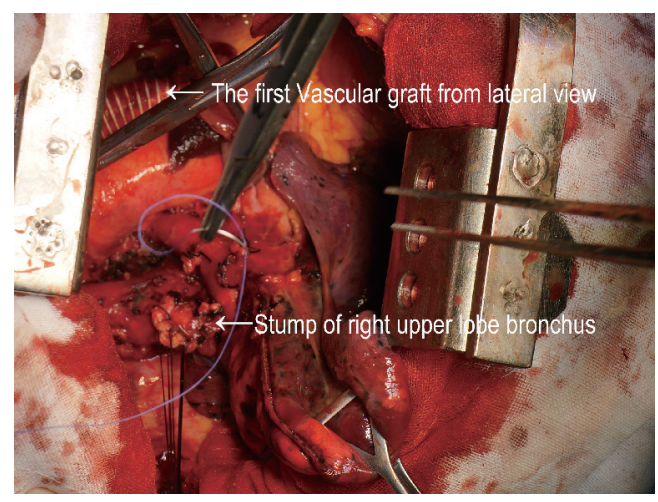

Figure 5 Demonstration of the second step via posterolateral thoracotomy: resection of the right upper lobe and systematic lymphadenectomy.

Then, at the second step, the patient was placed at left lateral decubitus position and underwent right posterolateral thoracotomy through the fourth intercostal space, via which radical lobectomy of the right upper lobe and systematic dissection of the right mediastinal lymph nodes was achieved (Figure 5). And the right brachiocephalic vein was freed and cut off, and then it was also reconnected to the root of the SVC (Figure 6) with another 12-mm GORE$\mathrm{TEX}^{\circledR}$ vascular graft using 4-0 prolene sutures (Johnson \& Johnson) for continuous suturing after complete resection of SVC. Finally, the incision of posterolateral thoracotomy was also closed up in a standard fashion. The total blood loss was about $1,000 \mathrm{~mL}$, and the patients were transfused with a total of $1,500 \mathrm{~mL}$ blood. The total operative time was about 6 hours for the whole surgery.

Postoperatively, the mass was pathologically and immunohistochemically diagnosed as lung adenocarcinoma (pT4N3M0, IIIC). The postoperative course for the patient was uneventful (Figure 7), and he was discharged at postoperative day 12. This patient received long-term oral administration of warfarin with a target of controlling the international normalized ratio (INR) to a range of 1.5-2.0. He was referred for adjuvant chemotherapy later. Now this patient has completed four cycles of adjuvant chemotherapy, and his recent chest CT (after 8 months) revealed no sign of recurrence.

\section{Results}

From January 2017 to March 2018, this two-step surgical approach combining median sternotomy and subsequent 
posterolateral thoracotomy was applied in 5 patients including the above typical case. Informed consent was obtained from all those patients. And our study was approved by the Ethics Committee of West China Hospital (No. 20170309). For preoperative evaluation, all patients underwent contrast-enhanced chest CT and upper abdominal CT, brain magnetic resonance imaging,
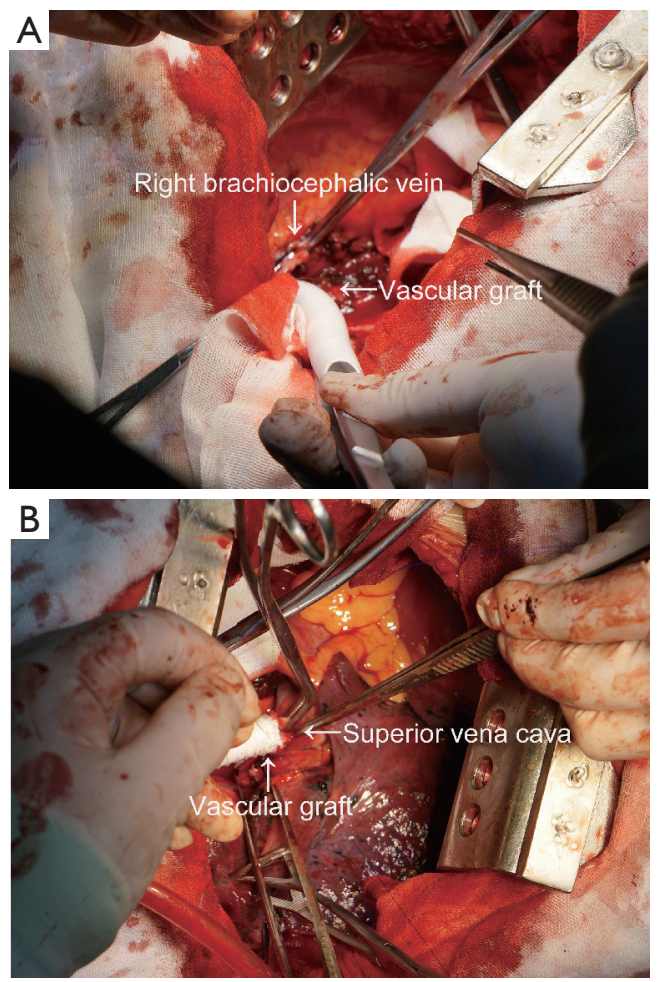

Figure 6 Demonstration of the second step via posterolateral thoracotomy: (A) anastomosis between right brachiocephalic vein and vascular graft; (B) anastomosis between vascular graft an superior vena cava. bone scanning, fiberoptic bronchoscopy examination, and cardiopulmonary function tests. The individual baseline characteristics of those patients were shown in Table 1. The median age of those patients was 34 years old (range, 17-65 years old). There were three male patients and two female patients. None of those patients had any comorbidity before surgery. All those patients had a locally advanced disease stage but their tumors were evaluated as technically resectable diseases with two-stage surgical approach combining median sternotomy with subsequent posterolateral thoracotomy. The preoperative INR values for all patients were normal while after surgery all of them maintained an INR value of 1.5-2.0 via oral administration of warfarin. Only one patient received preoperative chemotherapy because of the relatively advanced disease stage. The individual perioperative data of those patients were shown in Table 2. All those patients underwent at least one blood vessel reconstruction with lobectomy and systematic lymphadenectomy. And all patients achieved R0 resection. The median blood loss volume of those

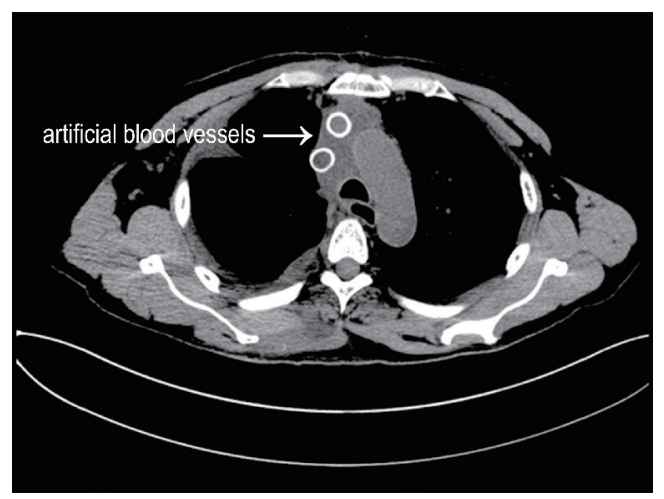

Figure 7 Chest computed tomography scan of the patient in postoperative day 10 showing the grafts patency.

Table 1 Individual baseline characteristics of those patients undergoing surgery via two-step surgical approach

\begin{tabular}{lccccccc}
\hline Patients & Age & Gender & Comorbidity & Preoperative stage & $\begin{array}{c}\text { Preoperative } \\
\text { INR value }\end{array}$ & $\begin{array}{c}\text { Postoperative } \\
\text { INR value }\end{array}$ & Preoperative treatment \\
\hline 1 & 42 & Male & None & cT4N3M0: IIIC & 0.97 & 1.52 & None \\
2 & 53 & Male & None & cT4N2M1a: IVA & 1.02 & 1.88 & Four cycles of chemotherapy \\
3 & 17 & Male & None & cT4N1M0: IIIA & 1.16 & 1.56 & None \\
4 & 34 & Female & None & cT4N0M0: IIIA & 0.89 & 1.77 & None \\
5 & 65 & Female & None & cT4N0M0: IIIA & 0.87 & 1.96 & None \\
\hline
\end{tabular}

INR, international normalized ratio. 


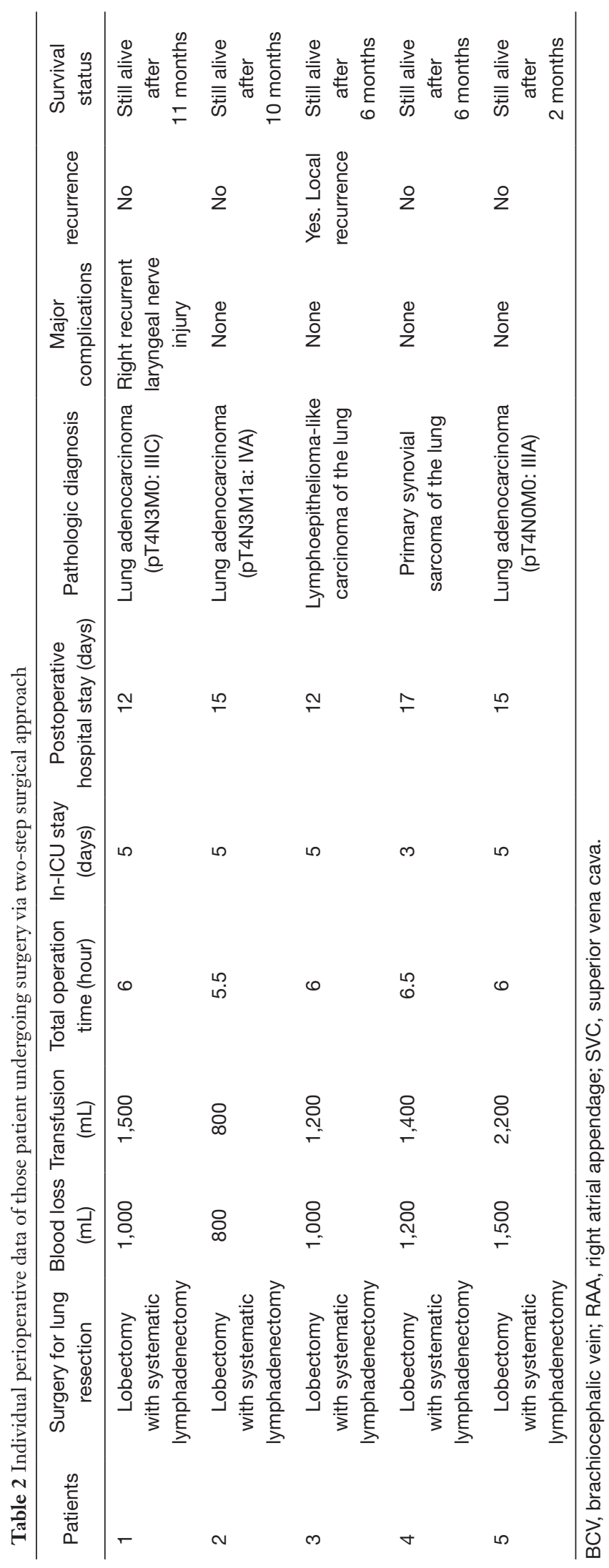

patients was $1,000 \mathrm{~mL}$ (range, $800-1,500 \mathrm{~mL}$ ) and the median transfusion volume of those patients was $1,400 \mathrm{~mL}$ (range, 800-2,200 mL). It took an average operation time of about 6 hours for the whole surgical procedure. Postoperatively, the median in-ICU stay of those patients was 5 days (range, 3-5 days) and the median in-hospital stay of those patients was 15 days (range, 12-17 days). Only one patient experienced right recurrent laryngeal nerve injury while other patients had no major complications. Moreover, none of those patients had rib fractures or postoperative functional disability.

All those patients underwent adjuvant therapy after surgery. We conducted our last follow-up in March 2018. All patients were alive and their recent CT scans showed graft patency rate was $100 \%$. Only one patient had locoregional recurrence at 3 months after surgery while other four patients had no sign of recurrence during follow-up.

\section{Discussion}

Lung cancers invading SVC with mediastinal lymph nodes metastasis were usually considered as contraindication of surgery (2). However, evidence has shown that such patients could benefit significantly from surgery if radical resection is achieved $(3,4)$. Patient with such locally advanced lung cancers undergoing surgical resection were reported to yield a 5 -year survival rate of $29-48 \%$ with acceptable postoperative morbidity and mortality rates $(5,9,10)$. For locally advanced lung cancers requiring both lung resection and SVC reconstruction, usually a hemi-clamshell approach was utilized for surgical resection. However, this approach has several disadvantages. First, it has the limitation of insufficient exposure of the posterior mediastinum which is required for systematic lymphadenectomy for lung resection, and hence, it is not feasible for patients with tumor or lymph node invasion of posterior mediastinum or with severe adhesions in the thoracic cavity. Second, it could cause rib fracture easily and lead to flailed chest. Third, this approach preserves sternoclavicular articulation and leads to decreased postoperative functional disability (7). Therefore, for locally advanced lung cancer involving both upper right lung and SVC, a hemi-clamshell approach is not sufficient for concomitant lobectomy with systematic lymphadenectomy and SVC reconstruction. In the preoperative evaluation, all those patients included in our study were carefully evaluated to have a technically resectable disease. Even though one patient was found to have enlarged contralateral mediastinal lymph nodes $(\mathrm{cN} 3)$, these lymph 
nodes could be radically resected because of no extracapsular invasion via median sternotomy. Another patient was found to have a solitary lung nodule at contralateral upper lung lobe, but the nodule was located peripherally near anterior mediastinum and could be resected through median sternotomy. Hence, we introduced a two-step surgical approach combining median sternotomy and subsequent posterolateral thoracotomy for such locally advanced lung cancers: the first step is median sternotomy via which the left brachiocephalic vein was freed and reconnected to the right atrial appendage and completed resection of contralateral mediastinal lymph nodes or solitary nodules could be achieved, and the second step is posterolateral thoracotomy via which lobectomy with systematic lymphadenectomy as well as reconnection of the right brachiocephalic vein to the right atrium could also be achieved.

This two-step surgical approach combining median sternotomy and subsequent posterolateral thoracotomy had several advantages. First, it combines the advantages of both sternotomy and thoracotomy and overcomes disadvantages of the two surgical approaches applied alone. Therefore, it has enough exposure for both anterior and posterior mediastinums for completing both lung resection with systematic lymphadenectomy and SVC reconstruction. Our five cases above have proven its excellent exposure allowing successful resection of the lung and reconstruction of SVC since all those patients achieved R0 resection via the twostep surgical approach. Second, it will not increase the risk of rib fracture as traditional hemi-clamshell approach. In our five cases, no patient experienced rib fracture during surgery. More importantly, our novel approach was proven to be safe since all those cases had an uneventful recovery from the surgery without any major complications postoperatively. Therefore, this two-step surgical approach was proved to be safe and feasible permitting both lung resection with systematic lymphadenectomy and SVC reconstruction for locally advanced lung cancers involving both right upper lung and SVC.

However, several limitations existed in this two-step surgical approach. First, there is no going back in the phase of posterolateral thoracotomy. Therefore, careful preoperative evaluation of the resectability of the tumor is mandatory. Second, we conducted this approach in a very limited sample size, which could decrease our overall validity of the results. Finally, in this study, lacking of longterm survival data, only short-term survival outcomes were reported as a result of a short follow-up time. Therefore, further studies about this two-step surgical approach with larger sample size and longer follow-up time are warranted.

\section{Conclusions}

This two-step surgical approach combining both median sternotomy and subsequent posterolateral thoracotomy could provide excellent exposure of both anterior and posterior mediastinums for easy concomitant lung resection with systematic lymphadenectomy and SVC reconstruction. This surgical approach was proved to be safe and feasible for locally advanced lung cancers involving both right upper lung and SVC.

\section{Acknowledgements}

Funding: This study was supported by the grant from the National Key Research and Development Project of China (to Q Zhou) (No. 2016YFE0103400).

\section{Footnote}

Conflicts of Interest: This study has been presented as oral presentation at the 26th Annual Meeting of the Asian Society for Cardiovascular and Thoracic Surgery (May 24-27, 2018, Moscow, Russia).

Ethical Statement: The study was approved by the Ethics Committee of West China Hospital (No. 20170309) and informed consent was obtained from all those patients.

\section{References}

1. Lee DS, Flores RM. Superior vena caval resection in lung cancer. Thorac Surg Clin 2014;24:441-7.

2. Tanaka $Y$, Hokka D, Ogawa H, et al. Surgery for malignant lesions of the chest which extensively involved the mediastinum, lung, and heart. Gen Thorac Cardiovasc Surg 2017;65:365-73.

3. Spaggiari L, Tessitore A, Casiraghi M, et al. Survival after extended resection for mediastinal advanced lung cancer: lessons learned on 167 consecutive cases. Ann Thorac Surg 2013;95:1717-25.

4. Dartevelle PG, Mitilian D, Fadel E. Extended surgery for T4 lung cancer: a 30 years' experience. Gen Thorac Cardiovasc Surg 2017;65:321-8.

5. Suzuki K, Asamura H, Watanabe S, et al. Combined resection of superior vena cava for lung carcinoma: 
prognostic significance of patterns of superior vena cava invasion. Ann Thorac Surg 2004;78:1184-9; discussion 1184-9.

6. Korst RJ, Burt ME. Cervicothoracic tumors: results of resection by the "hemi-clamshell" approach. J Thorac Cardiovasc Surg 1998;115:286-94; discussion 294-5.

7. Shintani Y, Kanzaki R, Kawamura T, et al. Surgical resection for advanced lung cancer using the hemiclamshell approach. Interact Cardiovasc Thorac Surg 2017;25:462-8.

8. Ohta M, Hirabayasi H, Shiono H, et al. Hemi-clamshell approach for advanced primary lung cancer. Thorac Cardiovasc Surg 2004;52:200-5.

9. Dartevelle PG, Chapelier AR, Pastorino U, et al. Longterm follow-up after prosthetic replacement of the superior vena cava combined with resection of mediastinalpulmonary malignant tumors. J Thorac Cardiovasc Surg 1991;102:259-65.

10. Spaggiari L, Regnard JF, Magdeleinat P, et al. Extended resections for bronchogenic carcinoma invading the superior vena cava system. Ann Thorac Surg 2000;69:233-6.

Cite this article as: Deng HY, Qin CL, Qiu XM, Tang XJ, Zhu DX, Zhou Q. A two-step surgical approach combining sternotomy and subsequent thoracotomy for locally advanced lung cancers requiring both right upper lung resection and superior vena cava reconstruction. J Thorac Dis 2018;10(8):4831-4837. doi: 10.21037/jtd.2018.07.77 